\title{
Comparative genomic analysis reveals the evolution and environmental adaptation strategies of vibrios
}

\author{
Heyu Lin $^{1 \dagger},{\text { Min } \mathrm{Yu}^{1 \dagger} \text {, Xiaolei Wang }}^{1}$ and Xiao-Hua Zhang ${ }^{1,2,3^{*}}$
}

\begin{abstract}
Background: Vibrios are among the most diverse and ecologically important marine bacteria, which have evolved many characteristics and lifestyles to occupy various niches. The relationship between genome features and environmental adaptation strategies is an essential part for understanding the ecological functions of vibrios in the marine system. The advent of complete genome sequencing technology has provided an important method of examining the genetic characteristics of vibrios on the genomic level.
\end{abstract}

Results: Two Vibrio genomes were sequenced and found to occupy many unique orthologues families which absent from the previously genes pool of the complete genomes of vibrios. Comparative genomics analysis found vibrios encompass a steady core-genome and tremendous pan-genome with substantial gene gain and horizontal gene transfer events in the evolutionary history. Evolutionary analysis based on the core-genome tree suggested that $V$. fischeri emerged $\sim 385$ million years ago, along with the occurrence of cephalopods and the flourish of fish. The relatively large genomes, the high number of $16 \mathrm{~S}$ rRNA gene copies, and the presence of R-M systems and CRISPR system help vibrios live in various marine environments. Chitin-degrading related genes are carried in nearly all the Vibrio genomes. The number of chitinase genes in vibrios has been extremely expanded compared to which in the most recent ancestor of the genus. The chitinase A genes were estimated to have evolved along with the genus, and have undergone significant purifying selective force to conserve the ancestral state.

Conclusions: Vibrios have experienced extremely genome expansion events during their evolutionary history, allowing them to develop various functions to spread globally. Despite their close phylogenetic relationships, vibrios were found to have a tremendous pan-genome with a steady core-genome, which indicates the highly plastic genome of the genus. Additionally, the existence of various chitin-degrading related genes and the expansion of chitinase $A$ in the genus demonstrate the importance of the chitin utilization for vibrios. Defensive systems in the Vibrio genomes may protect them from the invasion of external DNA. These genomic features investigated here provide a better knowledge of how the evolutionary process has forged Vibrio genomes to occupy various niches.

Keywords: Vibrio, Comparative genomics, Phylogeny, Environmental adaptation, Gene gain/loss, Chitinase

\footnotetext{
*Correspondence: xhzhang@ouc.edu.cn

${ }^{\dagger}$ Equal contributors

${ }^{1}$ College of Marine Life Sciences, Ocean University of China, 5 Yushan Road,

Qingdao 266003, People's Republic of China

${ }^{2}$ Laboratory for Marine Ecology and Environmental Science, Qingdao

National Laboratory for Marine Science and Technology, Qingdao 266071,

People's Republic of China

Full list of author information is available at the end of the article
} 


\section{Background}

Vibrios represent a large subgroup of the class Gammaproteobacteria and are Gram-negative, usually motile rods. Generally, members of the Vibrio species are mesophilic and chemoorganotrophic, and have a facultatively fermentative metabolism [1]. Vibrios are ubiquitously distributed in water columns of marine and estuarine water, marine sediments, and aquaculture settings worldwide [2]. It has been shown that vibrios are characterized by remarkable biodiversity, having evolved to develop complex lifestyles [3]. Many studies have indicated that vibrios have enormous biomass in the ocean, and comprises more than $10 \%$ of the aquatic culturable bacterial population [4-7]. To date, more than 110 species are recognized in the genus Vibrio, many of which have been identified in recent years.

As representative obligate heterotrophs, vibrios utilize a wide range of carbon sources for energy [8], an interesting feature of which is the utilization of chitin. Chitin is the most abundant polysaccharide in the ocean, and the second most abundant polysaccharide in nature after cellulose [9]. Chitin is composed of repeating units of the monomer $\mathrm{N}$-acetylglucosamine (GlcNAc), and this material is an abundant carbon-nitrogen-energy source for marine microorganisms. Cycling of chitin is mainly by chitinolytic bacteria [10]. Many Vibrio species, such as $V$. cholerae [10], $V$. harveyi [11] and $V$. furnissii [12], are able to use chitin as their sole carbon source. A group of enzymes is required for vibrios to successfully convert chitin to monomer [10], in which process chitinases function to hydrolyze chitin into small oligomers. Chitinases have been received much attention and were believed to play a central role in the chitin degrading processes by bacteria [13]. Many physiological characteristics of vibrios were associated with the capacity of chitin-degradation, including chemotaxis, cell division, biofilm formation and natural competence [14].

The genomic features of vibrios in the past decades have been well studied, and with the increasing number of sequenced species, there are growing number of reports which focus on the genus rather than on specific members of the genus. The diversity and phylogenetics of vibrios has long been a matter of interest, and multilocus sequence analysis (MLSA) and constructing supertrees using housekeeping genes from whole genome sequencings has been used in the analysis [15], which was believed to be the most powerful tool until alternative genome-based methods were developed. Sawabe et al. [16] have studied the evolutionary history of vibrios using 8 housekeeping genes, and suggested the method to be very efficient and reliable. While comprising a considerable pan-genome $[15,17]$, the genome plasticity of vibrios aided by horizontal gene transfer (HGT) was considered to be an important method of acquiring new genes and accelerating evolution [18], and this rapid change in the composition of the genomes mainly related to cell envelope modification and secondary metabolites, helping vibrios to succeed in competition with other organisms [19]. The wide range of metabolisms in vibrios, which facilitates them to adapt to a wide array of environmental conditions has drawn much attention, especially the capability of degrading chitin $[8,12]$. Nevertheless, although many studies mentioned above have been carried out to investigate the diversity, evolution and ecology of vibrios, many fundamental works are still needed in order to discover the underlying genetic basis, and this is now possible as the novel genomes and productive tools have recently become available.

For a long time, draft genomes have been employed to perform genomic studies. However, a complete sequence is better than partial genomes to examine all the genes or study the complete hereditary information of an organism [20]. The advent of the improved sensitivity and precision of complete genome sequencing technology provides a primary important path toward understanding the genome features of organisms. Lukjancenko et al. [17] noticed that the core gene numbers of Vibrio chromosome in complete genomes dropped sharply to only half of that when considering draft genomes, which was partly due to the incomplete information stored in the draft genomes. Besides, the rRNA operon number of draft Vibrio genomes is only one in most cases, whereas the complete genomes have seven or eight copies generally. Given the increased availability of complete genome sequences in the public databases and the development of comparative genomic analysis approaches, a more integrated and comprehensive view of the genus Vibrio becomes possible. There were more than 1500 genomes of vibrios available in the GenBank database at the time of this study, 18 of which were complete genomes sequences of the Vibrio species.

In this study, we sequenced two new complete Vibrio genomes, and presented a broad comparative analysis of these genomes across the genus with 18 other complete genomes of Vibrio spp. available in the GenBank database (http://www.ncbi.nlm.nih.gov). Firstly, the phylogeny of the genus Vibrio was inferred using coregenome. Secondly, the ancestral reconstruction and the estimation of emerge time of vibrios were performed to provide new knowledge about the evolutionary history of the genus. Thirdly, various environmental adaptation strategies, especially the ability of the chitin metabolism, were investigated to help understand how vibrios adapt to a variety of habitat niches. Finally, all the Vibrio genomes, including those draft sequences in the public database, along with a set of other representative marine bacteria, were employed to provide a comprehensive assessment of genome features. Our analysis helps in the 
understanding of the genetic characteristics of Vibrio and serves as a guide for further research.

\section{Methods}

\section{Bacterial strains}

The two strains $V$. rotiferianus B64D1 and $V$. mediterranei QT6D1 were isolated from bottom seawater of the Bohai Sea $\left(119.04^{\circ} \mathrm{E}, 38.23^{\circ} \mathrm{N}, 17.5 \mathrm{~m}\right.$ water depth) in August 2015 and the East China Sea $\left(122.0051^{\circ} \mathrm{E}, 26.8957^{\circ} \mathrm{N}\right.$, $92 \mathrm{~m}$ water depth) in October 2015, respectively. No animal ethics approval was required, and the field sampling procedures met local guidelines. Both strains were isolated using thiosulfate citrate bile salts sucrose (TCBS) agar (Hopebio, China) and demonstrated strong capacity to degrade chitin when growing on chitin agar plates. The strains were routinely grown aerobically on marine $2216 \mathrm{E}$ agar (MA; Becton Dickinson) at $28{ }^{\circ} \mathrm{C}$. Genomic DNA of the two strains were extracted using the phenol-chloroformisoamylic alcohol extraction protocol described by Marmur [21], and the 16S rRNA genes were sequenced to validate the strains.

\section{Genome sequencing and annotation}

Whole-genome shotgun sequencing of $V$. rotiferianus B64D1 and $V$. mediterranei QT6D1 was carried out with paired-end sequencing by HiSeq 4000 (Illumina) and long sequencing by PacBio RS II Sequencer (Pacific Biosciences, Menlo Park, CA). The long sequence was assembled using Canu v1.1 [22], and Pilon v1.16 [23] was used to polish PacBio assemblies according to Illumina data. Misassemblies were identified and corrected using REAPR [24] and manual inspection. Open reading frame (ORF) prediction and annotation were carried out using the RASTtk pipeline [25]. tRNA-encoding genes and rRNA operons were found by using tRNAscan [26] and RNAmmer [27], respectively. Restriction-modification (R-M) systems were identified by searching the RAST annotation, and CRISPRfinder [28] was used to search for CRISPR (clustered regularly interspaced short palindromic repeats) regions. When the CRISPR regions was recognized as questionable CRISPRs, the presence of Cas (CRISPR-associated protein) genes was considered evidence for a functional CRISPR system.

The two complete genomes sequenced in this study and a further 18 complete genomes of representative strains in distinct species of the genus Vibrio were collected together as the "small dataset" (Additional file 1: Table S1), while all the 1582 Vibrio genomes available in GenBank except those with low qualities (Contig N50 <10,000 bp) were retrieved to make up the "large dataset". Vibrio species included in the small dataset were isolated from various sources, such as oceans, estuarine environments, humans, marine fishes and marine molluscs, which enabled the provision of a holistic profile of the genus. In particular, V. fischeri (Allivibrio fischeri) and V. damselae (Photobacterium damselae subsp. damselae) had been considered involved in the genus Vibrio for a long time. Although they have been reclassified as other closely related genus $[29,30]$, we decided to involve them in this study to acquire richer information on the evolution and forces for genome changes of the group due to their relatively unique living strategies.

\section{Pan/core genomes and phylogenetic analysis}

The program GET_HOMOLOGUES v3.0.3 [31], with three popular clustering algorithms, i.e. bidirectional best hit (BDBH), COGtriangles [32] and OrthoMCL [33], was used for clustering orthologous genes and identifying core- and pan-genomes within the small dataset mentioned above under default parameter values. Sequences of all the single-copy orthologues were aligned individually using the MAFFT software with the "linsi" algorithm [34], and the alignments were concatenated into one extended alignment. Subsequently, TrimAl v1.2 [35] was applied to remove ambiguous and unreliable fragments from the concatenated alignment with the parameter "-gt 1". The phylogenetic relationship of the concatenated data was inferred using RAxMLv8.2.4 [36] based on the Maximum-likelihood (ML) algorithm with the PROTGAMMALGF evolutionary model and 1000 bootstrap replicates, after Prottest 3.4 [37] was performed to estimate the best-fit substitution model. The genome of Shewanella denitrificans OS217 was chosen to serve as an outgroup. The phylogenetic network of the concatenated data was constructed using SplitsTree v4.14.6, with a neighbor net drawing and Jukes-Cantor correction [38]. A pangenome tree was constructed according to the presence/ absence of all homologous genes using RAxML. Besides, we identified the specific genome (unique gene pool of a species) of the two new strains we sequenced in this study among the noncore region, in order to investigate the unique properties of them.

A chronogram for 23 taxa RAxML phylogeny was constructed using the method of penalized likelihood [39], implemented in r8s v1.81 [40]. The estimated origin time of cyanobacteria (2700 3500 million years ago, MYA) [41] and the divergence time between Escherichia coli and Salmonella enterica (140 MYA) [42] were used as calibration points. The reference ML tree was built based on 332 single-copy core orthologues shared among all the 23 bacterial species. The optimal smoothing parameter was determined through a cross-validation procedure that involved pruning terminal branches.

\section{Reconstruction of gene gain and loss events}

Reconstruction of ancestral gene content and the gene gain and loss during the evolution of the small dataset 
was conducted using the phylogenetic gain-loss-duplication model employed in the program COUNT [43]. The number of paralogs in each genome for all the identified gene families was taken into account in the reconstruction, and the topology of the core-genome tree was used as the reference tree. All the genes acquired or lost during the evolutionary process of the genus Vibrio were categorized based on searching against the COG database (http://www.ncbi.nih.gov/COG/) using RPS-BLAST program. SIGI-HMM [44] was used to infer whether genes in the genomes were acquired through HGT.

\section{Average nucleotide identity (ANI) calculation}

ANI between any two genomes of the small dataset was calculated using a custom Perl script adapted from ANI.pl (https://github.com/jhbadger/scripts/blob/master/ANI.pl), following the algorithm described by Goris et al. [45], and the resulted matrix was clustered and visualized using $R$ packages pheatmap software [46].

\section{Analysis of genes related to chitin degradation}

Functional proteins related to chitin metabolism in all annotated genomes were determined according to the chitin pathway defined for $V$. cholerae [8] and by being searched against all the annotations. Among these predicted chitinolytic enzymes, the evolutionary history of chitinase $\mathrm{A}$ in the small dataset was inferred using the Neighbor-Joining method [47] implemented in MEGA7 [48], and the phylogenetic tree was further tested for the selection pressure among amino acid sites using several codon-based ML procedures as implemented in the codeml tool of PAML v4.9 [49]. The conserved domains of chitinase A were defined by the NCBI batch CDsearch online tool (https://www.ncbi.nlm.nih.gov/Structure/bwrpsb/bwrpsb.cgi).

\section{Genomic comparison with other marine bacteria}

Thirteen complete genomes of common marine bacteria (Additional file 2: Table S2) were retrieved from GenBank to make comparisons with Vibrio genomes. These genomes are from four different classes including Cyanobacteria, Alphaproteobacteria, Gammaproteobacteria, and Flavobacteria, which are considered to account for up to $80 \%$ of the total bacterioplankton in the ocean [50]. Basic features of these 13 genomes, including genome sizes, 16S rRNA gene copy numbers, the number of genes derived from HGT process and genes encoding chitinase, were inferred according to the same methods as for the Vibrio species described above.

\section{Database accession numbers}

The complete genome sequences of $V$. rotiferianus B64D1 and V. mediterranei QT6D1 have been deposited in NCBI GenBank server under the accession number
CP018311 to CP018312 and CP018308 to CP018310, respectively.

\section{Results \\ General features of Vibrio species}

The general information of the 20 complete Vibrio genomes is summarized in Table S1. Each genome contains two chromosomes, and some species have one or more plasmids. The genome size of vibrios ranges from 4.09 to $6.32 \mathrm{Mb}$, with a mean size of $5.14 \mathrm{Mb}$. Most the other marine bacteria compared in this study have a lower genome size (3.62 $\mathrm{Mb}$ on average) compared to vibrios (Fig. 1a). Vibrios have a wide range of $\mathrm{G}+\mathrm{C}$ content, ranging from $38.3 \%$ in $V$. fischeri to $50.7 \%$ in $V$. furnissii. The ORF number and orthologue number range from 3807 to 5920 and 3669 to 5593, respectively. Strikingly, vibrios contain a much higher number of $16 \mathrm{~S}$ rRNA gene copies (7-15) than most of the other marine bacteria (3 on average) used for comparison in our study (Fig. 1b), which may reflect the capability of rapid growth of Vibrio species. In addition, the genome size of the large dataset ranges from 2.9 to 6.7 $\mathrm{Mb}$, and the $\mathrm{G}+\mathrm{C}$ content ranges from $38.0 \%$ to

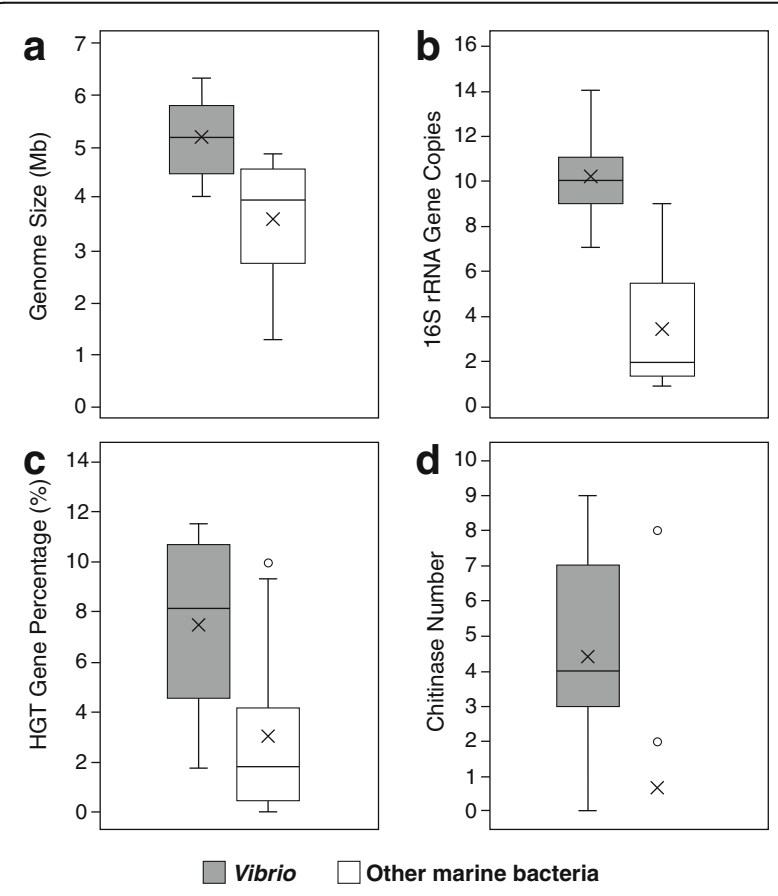

Fig. 1 Comparison of genomic characteristics between the 20 vibrios with complete genomes and other representative marine bacteria. Box-and-whisker plot showing data distribution and interquartile ranges for each group with the median value displayed as a line in each box plot. Outliers are shown as circles, and mean values are shown as saltires in the figure. a Comparison of the genome sizes. $\mathbf{b}$ Comparison of the copy numbers of 165 rRNA gene. $\mathbf{c}$ Comparison of the percentage of HGT genes. $\mathbf{d}$ Comparison of the number of chitinase coding genes 
$57.2 \%$, which suggests a higher diversity of Vibrio genomes. However, due to the missing information of those draft genomes, there is some bias definitely existing in the large dataset.

\section{R-M and CRISPR systems}

Microbes rely on diverse defense mechanisms that protect them from mobile elements or foreign DNA implicated in the reduction of HGT. The common bacterial defense systems include R-M systems and CRISPR systems [51]. We identified these two defense systems in the small dataset (Additional file 1: Table S1), and found that almost all the Vibrio genomes contained the R-M system except for $V$. breoganii FF50 and $V$. tritonius JCM 16456. Specifically, all the vibrios containing R-M systems have type I R-M system, while type II R-M system was additionally harbored by $V$. cholerae KW3, $V$. rotiferianus B64D1, $V$. tubiashii ATCC 19109 and $V$. vulnificus FORC 017, and type III R-M system was additionally harbored by $V$. coralliilyticus RE98. Eight out of the 20 genomes had CRISPR systems (Additional file 1: Table S1), while the cas gene numbers (1 to 8 ) and the lengths of direct repeats (DRs) of each CRISPR (23 to 51) were distinct from each genome. The identification of DRs in the CRISPR would be quite difficult if performed in draft genomes, since the short and repetitive sequencing reads would be difficult to assembly correctly.

\section{Core and pan-genome}

To determine the global gene repertoire and the genes conserved across all the vibrios, the power law model of Tettelin et al. [52] was used to describe the pan- and core-genomes based on the 20 complete Vibrio genomes. All the genes were grouped into 21,844 families, which represented the Vibrio pan-genome. The progression of the pan- and core-genomes after ten random samplings was calculated (Additional file 3: Figure S1). The number of novel gene families in the pan-genome gradually increased with no limitation when more genomes were considered, leading to an open pangenome. Nevertheless, the number of conserved gene families constituting the core genome decreased slightly, and the extrapolation of the curve indicated that the core genome reached a minimum of 1630, and would remain relatively constant, even as many more genomes were added.

\section{Specific genomes of V. rotiferianus B64D1 and V. mediterranei QT6D1}

To investigate the unique characters of the two complete genomes sequenced by us, we identified the specific genome of each genome, i.e., orthologous families that contain genes exist in each of these two genomes but absent in other 18 previously sequenced genomes. In the total
21,844 pan-genome families, 397 specific genes were possessed by $V$. rotiferianus $\mathrm{B} 64 \mathrm{D} 1$, whereas 871 were possessed by $V$. mediterranei QT6D1. The COG functional annotation of the specific genomes (Additional file 4: Figure S2) showed that genes related to carbohydrate transport and metabolism (G) and transcription (K) are extremely abundant (53 and 40, respectively) in $V$. mediterranei QT6D1, while the most abundant COG category in the specific genome of $V$. rotiferianus B64D1 is general function prediction only $(\mathrm{R})$ and cell wall $/ \mathrm{mem}$ brane/envelope biogenesis (M). Furthermore, it is notable that there is a chitinase gene (BSZ05_18380) annotated by the RAST server involved in the specific genome of $V$. mediterranei QT6D1, which may contribute to its strong degradation capability for chitin.

Phylogenetic and divergence times analysis of vibrio species To investigate the phylogenetic relationship of Vibrio species, a robust core-genome tree was inferred based on the concatenated 1003 single-copy orthologues proteins shared by all the 20 complete genomes as shown in Fig. 2a. Two main clades could be determined in the tree, and these two clades could be further proved by the split network analysis (Fig. 2b). Clade I consisted of five species: $V$. harveyi, $V$. campbellii, $V$. rotiferianus, $V$. alginolyticus and $V$. parahaemolyticus, which was the so-called Vibrio core group [53] or Harveyi clade, and Clade II consisted of $V$. cholerae, $V$. mimicus, $V$. fluvialis and $V$. furnissii, which formed the Cholerae clade proposed by Sawabe et al. [54]. The pan-genome tree (Additional file 5: Figure S3) also had a relatively high bootstrap values at most of the nodes similar to the core-genome tree. However, the topologies of the panand core-genome trees were different, which might be attributed to the dispensable genes. The phylogenetic tree based on 16S rRNA genes was also inferred (Additional file 6: Figure S4), whereas it was not as reliable as the core-genome tree insofar as the bootstrap values were extremely low.

To explore the divergence times of vibrios, several calibration points were included in the analysis. The most recent common ancestor (MRCA) of vibrios (i.e., the emergence time of $V$. damsela) was estimated to have emerged around 536 MYA, whereas $V$. fischeri was estimated to have emerged around 385 MYA (Fig. 3).

\section{ANI values}

ANI values calculated through pairwise comparison of the 20 Vibrio genomes further elucidated their genetic relatedness. ANI has been considered the best approach to measure genetic distance between genomes, as it represents a mean of identity/similarity values between homologous genomic regions shared by two genomes, which enables minimization of bias brought about by 

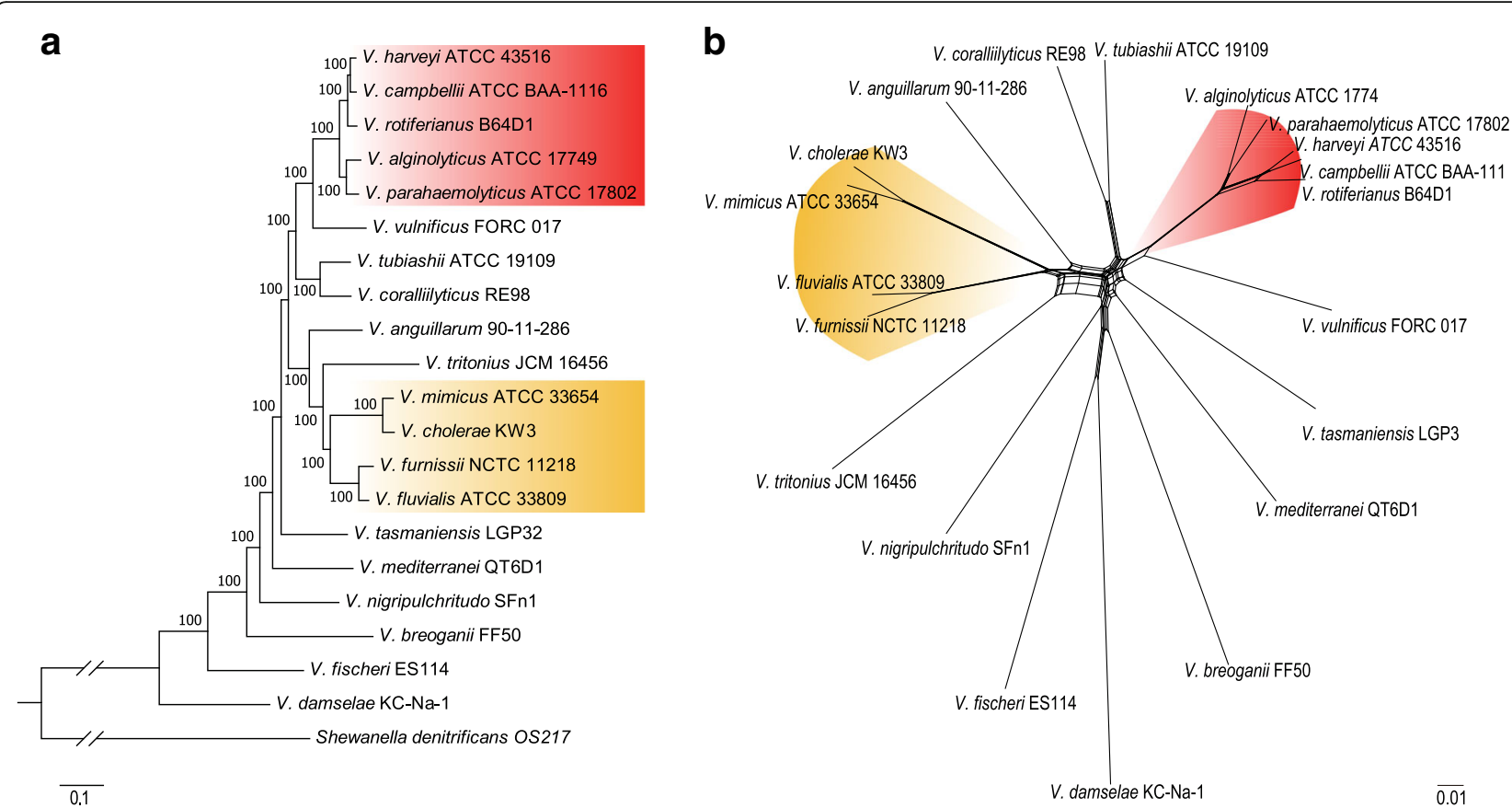

Fig. 2 Phylogenetic relationships of the 20 vibrios with complete genomes. The Harveyi clade and the Cholerae clade are highlighted in red and yellow respectively. a Core-genome tree reconstructed by RAxML software. The tree is rooted using $\mathbf{S}$. denitrificans OS217. The number at each node denotes the bootstrap value based on 1000 replicates, and the scale bar indicates the number of substitutions per site. b Phylogenetic network reconstructed by SplitsTree4 software

variable evolutionary rates and HGT events [55]. It was demonstrated that ANI values within a genus were generally higher than $62 \%$, and $95-96 \%$ ANI has been recommended as the delimitation criterion for different species [56]. The ANI values between Vibrio species ranged from $70 \%$ to $88 \%$, which was within the range of the classical boundary for a genus. Species groups could be derived after clustering according to the rows (Additional file 7: Figure S5), among which the Harveyi clade and Cholerae clade were consistent with the coregenome tree (Fig. 2a). Members of the Harveyi clade shared the highest ANI across the genus ranging from $80 \%$ to $88 \%$, indicating extremely close genetic relatedness within the Harveyi clade. Furthermore, the clades possessing high ANI values could serve as an explanation for the relatively short terminal branch lengths of their members in the phylogenetic tree, as the ANI represented the genetic distances between genomes.

\section{Gene gain and loss}

To gain insight into the origin and the variations of Vibrio genomes, we investigated the gene gain/loss events that might have occurred during the evolution of the genus Vibrio. The gene family numbers showed a noticeable upward trend during the evolutionary history, and the first time that gene loss events exceeded gene gain events was present at the MRCA of $V$. anguillarum
90-11-286 and V. mimicus ATCC 33654 (Fig. 4). This internal node led to a cluster that was mainly comprised by the Cholerae clade, and all the members at terminal nodes in this cluster had relatively small orthologues families $(<4600)$, which may be explained by the fact that gene loss events posed more significant impact on this cluster in the evolutionary process. On the contrary, other members in this phylogenetic tree experienced more and continuous gene gain events. However, no matter whether the species have suffered more gene loss events in a few internal nodes, the gene gain events in the evolutionary process were more numerous than the gene loss events in most instances, suggesting that vibrios have experienced remarkable gene family expansion in the evolutionary history. This generally expansive trend made the orthologue number of vibrios increase by approximately $50 \%$, i.e., from 2828 gene families in MRCA of vibrios to 4547 gene families on average as currently predicted (Additional file 1: Table S1). According to the COG functional annotation (Fig. 5) of the gain/loss genes, other than the poorly characterized genes (general function prediction only and function unknown, COG categories $\mathrm{R}$ and $\mathrm{S}$ ), genes related to transcription (K) formed the most abundant category of gene gains, followed by genes involved in carbohydrate transport and metabolism (G), whereas the most abundant category of gene losses was associated with signal 


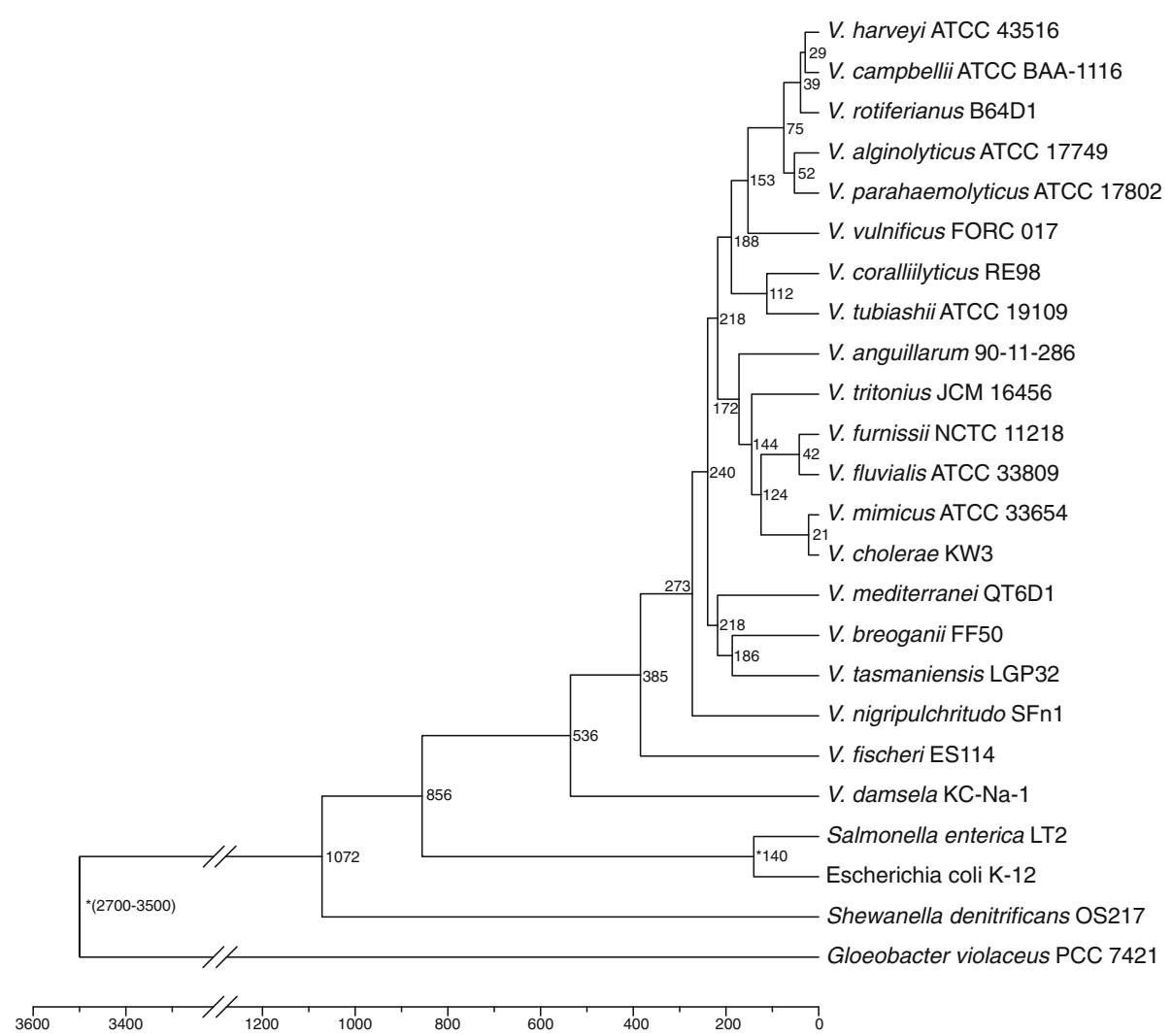

Fig. 3 Chronogram of the 20 vibrios with complete genomes. The value near each internal branch is the estimated emerge time for that branch. Nodes with fossil record corrections are indicated with an asterisk

transduction mechanisms $(\mathrm{T})$. Gene gains were present in every COG category except for extracellular structures $(\mathrm{W})$ and nuclear structure $(\mathrm{Y}$, not applicable to prokaryotic COGs), whereas gene losses in the history were not present in RNA processing and modification (A), extracellular structures (W), nuclear structure (Y) and cytoskeleton ( $\mathrm{Z}$, not applicable to prokaryotic COGs).

HGT is a significant driver of gene innovation in the life of bacteria [57]. A search for genes with aberrant codon usage statistics was carried out to predict possible genomic islands, referred to as alien genes. Surprisingly, the average percentage of alien genes among these vibrios was predicted to be $7.3 \%$, with some species containing up to $10 \%$ alien genes. The class Gammaproteobacteria was the major donor of HGTs, contributing as much as $72.1 \%$ alien genes, followed by Sphingobacteria, which contributed 6.5\% of the alien genes. This percentage of alien genes was significantly higher than that of the other compared marine bacteria, with nearly all of the genomes only involved having no more than 5\% alien genes, except for species from Marinobacter and Rhodobacter, which had $9.96 \%$ and $9.25 \%$ alien genes, respectively (Fig. 1c).

\section{Chitin degradation related genes}

Many Vibrio species have been reported as capable of degrading chitin. Chitin degradation is achieved by a complex pathway including multiple chitinases [58]. Seventeen different protein families related to chitin metabolism were retrieved from Vibrio genomes (Additional file 8: Figure S6). Eighteen out of the 20 vibrios (except for $V$. breoganii FF50 and $V$. tritonius JCM 16456) contained chitinase (EC 3.2.1.14), and all the 20 vibrios contain $\beta$ - $\mathrm{N}$-acetylhexosaminidase (EC 3.2.1.52). Moreover, $\sim 99 \%$ of the genomes in the large dataset carried both these two genes, which have the ability to completely hydrolyze chitin to monomer GlcNAc [59]. In contrast to this abundance, these genes seldom exist in the compared marine bacteria, except for strains from genus Pseudoalteromonas and Shewanella, which carried eight and two chitinase encoding genes in their genomes, respectively (Fig. 1d).

Chitinases distributed in these 18 Vibrio species come from 15 protein families, which can be classified into four categories (Fig. 6). Class I encompasses a family GH18 chitinase domain, while Class II possesses a similar chitinase domain as Class I but with an insertion domain in it. Class III encompasses a family GH19 chitinase domain, which was considered to be rare in 


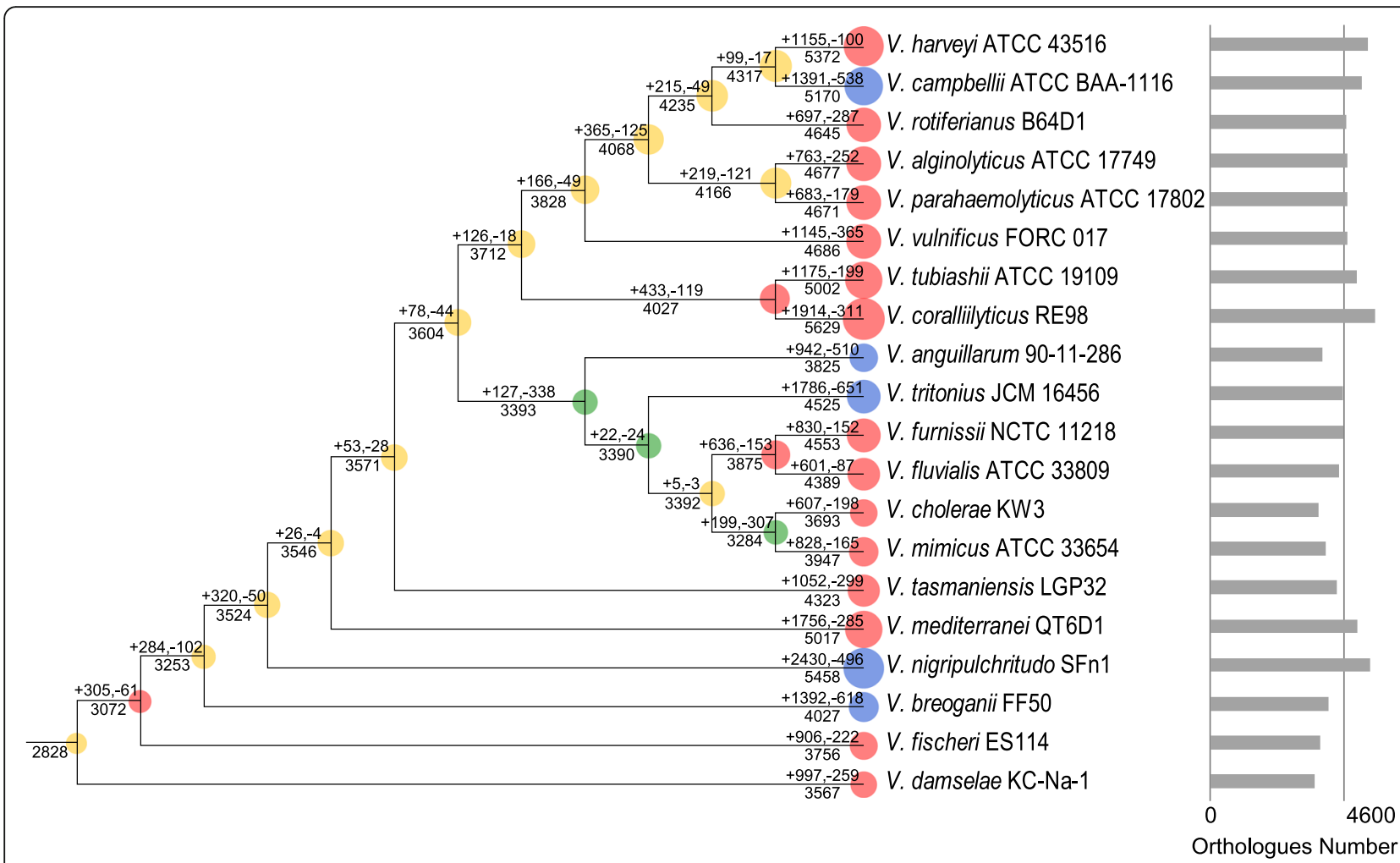

Fig. 4 Ancestral genome content reconstruction of the 20 vibrios with complete genomes. The numbers of gene gain and loss are shown above each branch, and the numbers of gene present at each node are shown below the corresponding branch. The area of the circle at each node indicate the genome size of the node. Nodes with more gene gain events (net gains > net losses) are indicated in yellow; Nodes with more gene loss events (net losses $>$ net gains) are indicated in green; Nodes with significant genome expansions (>10\% net gains) are indicated in red; Nodes with significant genome expansions and reductions at the same time (>10\% net gains and $>10 \%$ net losses) are indicated in blue. The bar chat on the right indicates the orthologues number of each terminal node

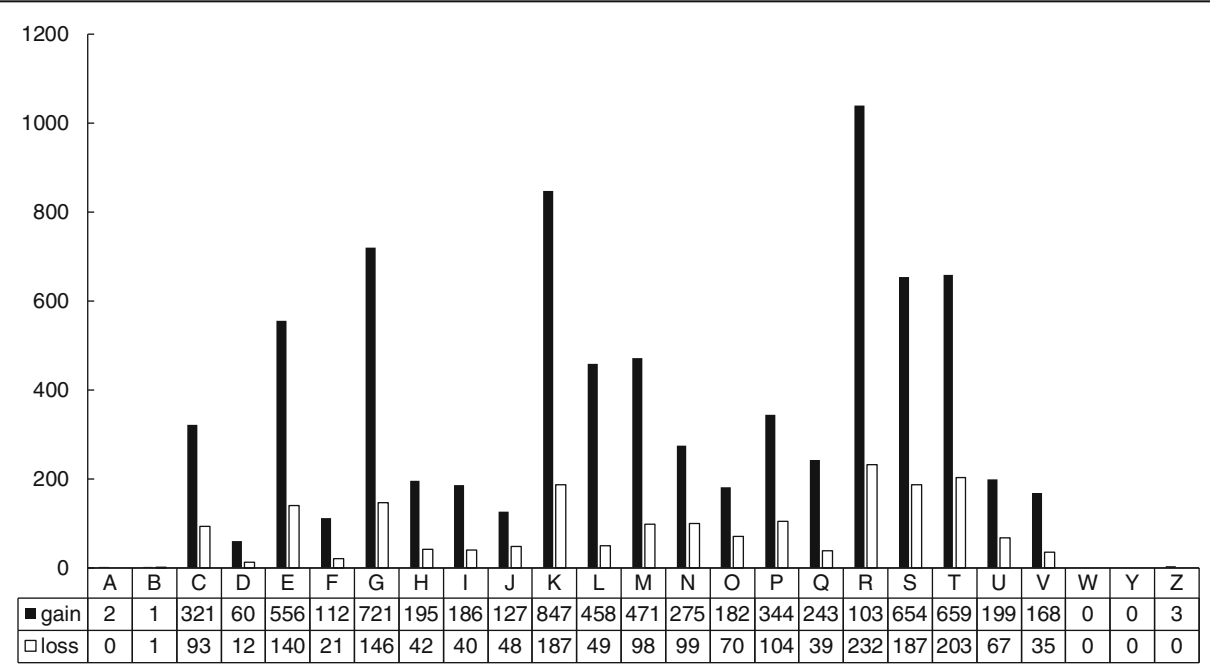

Fig. 5 COGs classification of putative gene gains and losses occurred in the evolutionary history of vibrios. Designations of functional categories: [A] RNA processing and modification, [B] chromatin structure and dynamics, $[\mathrm{C}]$ energy production and conversion, [D] cell cycle control and mitosis, $[\mathrm{E}]$ amino acid metabolism and transport, [F] nucleotide metabolism and transport, [G] carbohydrate metabolism and transport, [H] coenzyme metabolism, []] lipid metabolism, []] translation, [K] transcription, [L] replication and repair, [M] cell wall/membrane/envelope biogenesis, [N] Cell motility, [O] post-translational modification, protein turnover, chaperone functions, [P] Inorganic ion transport and metabolism, [Q] secondary metabolites biosynthesis, transport and catabolism, [R] general functional prediction only, [S] function unknown, $\mathrm{T}$ ] signal transduction, [U] intracellular trafficking and secretion, [V] Defense mechanisms, [W] Extracellular structures, [Y] nuclear structure, [Z] cytoskeleton 


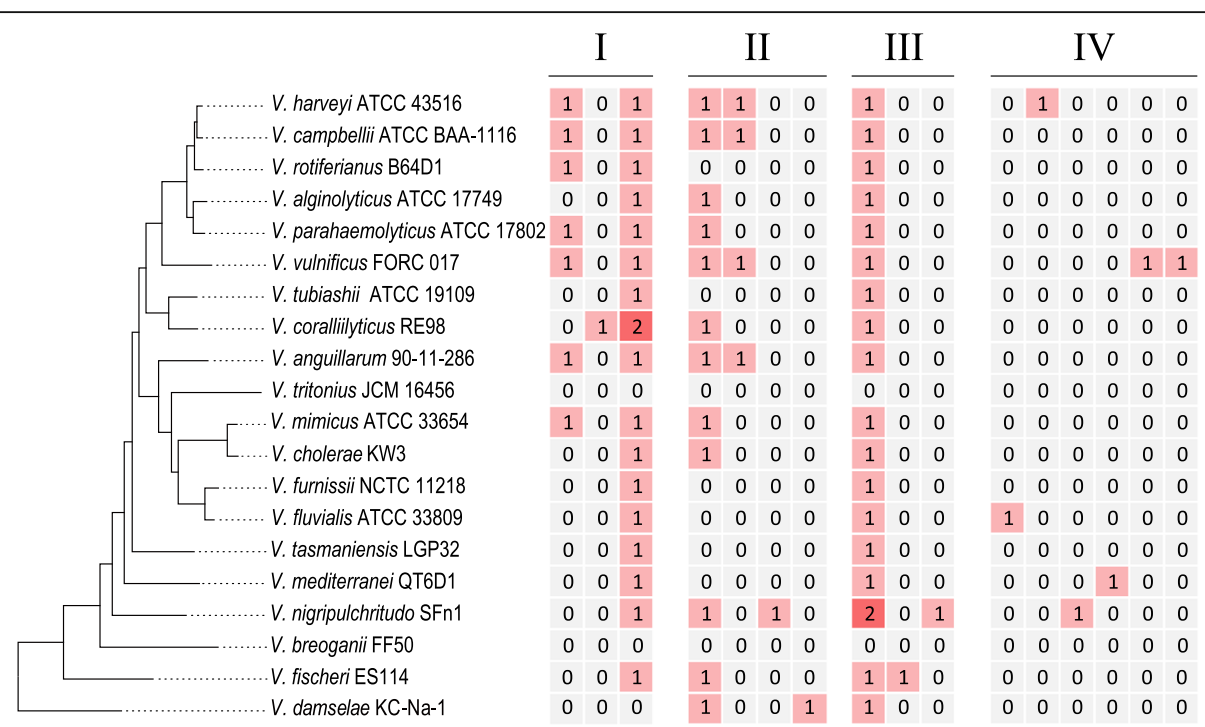

Fig. 6 Distribution of Subgroups of enzyme Chitinase involved in the 20 Vibrio species with complete genomes. Each of the columns corresponds to a chitinase cluster. The number within the box indicates the copy numbers of that gene cluster. Class I contains a family 18 chitinase domain; Class II contains a family 18 chitinase domain with a chitinase insertion domain; Class III contains a family 19 chitinase domain; Class IV contains no significant chitinase domain based on NCBI conserved domain database. Columns belonged to the same class indicate they share similar component patterns of structure domains but failed to be clustered into the same protein family

bacteria [60]. However, all the 18 Vibrio genomes carried GH19 chitinase according to our analysis, indicating that GH19 chitinase is abundant in vibrios. Class IV does not contain any typical chitinase domain, but could be annotated as chitinase using blastp against the $\mathrm{nr}$ database (ftp:// ftp.ncbi.nlm.nih.gov/blast/db/), which may result from the relatively low conservation of these chitinases. Most studies on diversity of chitin-degrading enzymes in aquatic environments focused on chitinase A, which belonged to Class I chitinase mentioned above, since this enzyme was thought to be conserved in Proteobacteria and may be a potential indicator of chitinoclastic ability [8]. Gene chiA was present in 17 species of the genus Vibrio, almost all of which were single-copy, except $V$. coralliilyticus, which has two different copies of chiA. The phylogenetic tree of 18 chiA genes was constructed as shown in Fig. 7. The topology of the chiA tree was very similar to that of the core-genome tree (Fig. 2a), indicating the chiA gene evolved along with the Vibrio genus. Intriguingly, one of the two copies of $V$. coralliilyticus was shown to have a relatively distant relationship with other orthologues, suggesting its different evolutionary origin. Furthermore, there were more synonymous (Ks) changes than non-synonymous (Ka) changes in the evolutionary process of chiA, which indicated the chiA gene had been subjected to significant purifying (negative) selection.

\section{Discussion}

Genomic characters of $\mathrm{V}$. rotiferianus B64D1 and V. mediterranei QT6D1

The specific genome of an organism among a related group represent its unique character. We identified the specific genome of each of the two complete genomes we sequenced and found they occupied many unique orthologues families which absent from the previously genes pool of the complete genomes of vibrios. V. rotiferianus B64D1 comprised many unique genes related to

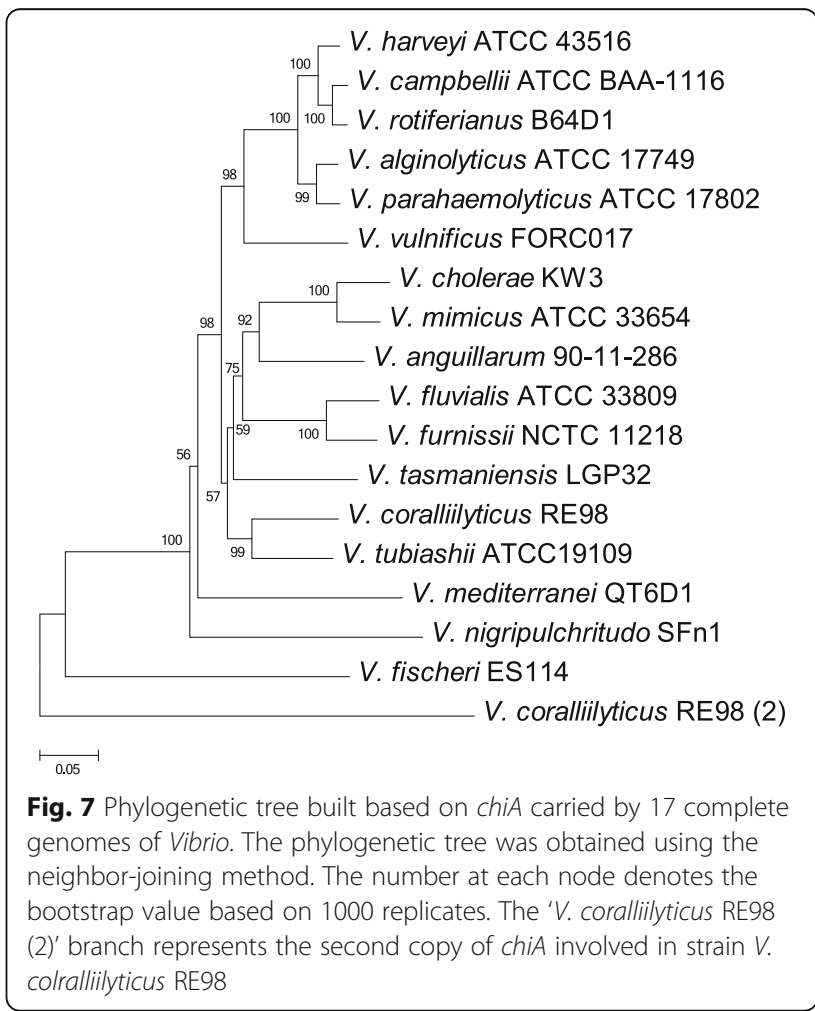


cell wall/membrane/envelope biogenesis (COG category $\mathrm{M})$, e.g., six orthologues families were annotated as glycosyltransferase, which may play an important role as the first level of protection against environmental stress. Up to 53 orthologues families in the specific genome of $V$. mediterranei QT6D1 were assigned into the COG category of carbohydrate transport and metabolism (G), among which 6 orthologues families were annotated as the component of ABC-type sugar transport system, which may indicate the potential specific ability for $V$. mediterranei QT6D1 to utilize different kinds of sugar in ambient environments. Besides, 46 orthologues families in the specific genome of $V$. mediterranei QT6D1 were assigned into the COG category of transcription (K), and 31 out of these 46 families were transcriptional regulator. The abundant unique transcriptional regulators present in the genome of $V$. mediterranei QT6D1 could enable it to regulate metabolic processes to adapt complex habitats. Furthermore, it is interesting that $V$. mediterranei QT6D1 carried a putative chitinase gene in its specific genome, which might explain the high activity to degrade chitin showed by the strain in our chitin degradation study.

\section{Genomics and the evolutionary dynamics of Vibrio species}

The genus Vibrio encompasses one of the most diverse group of heterotrophic marine bacteria [61, 62]. We investigated 20 Vibrio species with complete genomes (18 species were from the GenBank and two were from this study) and all the 1582 available draft Vibrio genomes in the GenBank. A set of common marine bacterial species which are widely distributed in the ocean was employed to make comparisons with vibrios. The numbers of predicted ORFs in Vibrio species differed from $\sim 3800$ to $\sim 6000$. Given the close phylogenetic relationship of these bacteria according to the ANI analysis, such a difference suggested substantial gene loss and/or gain in their evolution. Variable gene composition was the primary mode of adaptive evolution in microorganisms, leading to the enhancement of their physiological and ecological capabilities [63]. We therefore performed ancestral state reconstruction of vibrios and found generally expansion of the orthologues number throughout the whole evolutionary history of this group. Genome expansion evidence has been found in a few other groups of marine bacterioplankton, such as the genus Glaciecola [64], whereas the genome reduction (also known as the genome streamlining) has been reported in groups such as SAR11 [65] and the genus Prochlorococcus [66]. Many putative gene gains belonged to the function of transcription and carbohydrate transport and metabolism, which enabled vibrios to quickly response to environmental changes and use many different kinds of substrates to survive under various conditions. The function of signal transduction mechanisms has experienced the most gene loss, despite it has also experienced more than three times of gene gain in the evolution. The constantly changing of the components of this function may indicate vibrios absorb and discard genes as needed, in response to their environments. The massive genome content changes helped vibrios succeed within a niche, and the process of HGT can promote their evolution. We found that the percentage of alien genes in Vibrio genomes was significantly more than most of the other compared marine bacteria, suggesting that alien genes provided vibrios with a genetic basis of diversity and helped them to survive under their respective niche conditions. In addition, the HGT events appear to occur among bacteria in close relationship, as more than $70 \%$ alien genes of vibrios were estimated to come from the same class Gammaproteobacteria.

Bacteria have to change their genomes to adapt to variable conditions, and greater niche diversity will require larger pan-genomes. Given the varying marine environments in which the species distributed, it was understandable that vibrios had a large amount of pangenome contents, and this feature was in accordance with previous reports of some other marine bacteria such as the genus Shewanella [67] and Glaciecola [64]. In addition, the pan-genome tree was inferred based on homologous genes distributed in the whole-genome scales. The taxa relationships in the pan-genome tree differed from that in the core-genome tree, because the pan-genome tree focused on those genes that were not present in every genome, while on the contrary the core-genome tree focused on those genes that were present in all the genomes. Such a substantial difference between the two trees indicated that the dispensable genes, which may contain genes obtain from the HGT processes, played a crucial role in vibrios' genomic composition and evolution.

\section{Phylogeny and divergence times of Vibrio species}

Vibrio species have considerable biodiversity and extremely close phylogenic relationships within the genus [16]. 16S rRNA gene sequences could only provide a relatively low discriminating power to elucidate the evolutionary relationship of the group. The availability of complete genomes of Vibrio species enables a more definitive analysis of their relationships. Though highly plastic genomes may suffer lots of recombination and HGT events, it has been proved that the phylogenetic signal was still very strong within the core-genome [68]. We constructed a core-genome tree of the genus Vibrio from concatenated proteins of all the homologues shared by every species, and the result showed improved resolution and enormously increased robustness of phylogenetic analyses with nearly all the nodes having the bootstrap value of 100 . The availability of a robust 
phylogeny of the genus Vibrio enabled a reliable basis to conduct the following and future analysis.

The species $V$. fischeri might have occurred at 385 MYA, corresponding to the Devonian period ( 419.2-358.9 MYA). This was a period that the cephalopods (the order which squid belong to) has been estimated to divergent [69]. Besides, fish reached substantial diversity during this time, and many ray-finned fishes (Actinopterygii) occurred during the Devonian period [70]. Based on the phylogenetic tree, $V$. fischeri was a early member of vibrios to emerge, and it is a marine luminous bacterium that lives symbiotically with squids and fish [71]. Therefore, we could deduce that the rise of vibrios might have coincided with the occurrence of cephalopods and the flourishing of fish.

\section{Environmental adaptation strategies of vibrios}

Free-living bacteria encompass large genomes in most cases [72], and vibrios occupy relatively large genomes compared to most other common marine bacteria in this study, which enable them to search for resources (chemotaxis), change strategies to exploit patchy nutrients and target metabolites such as complex polysaccharides, which require complex enzyme repertoires.

The capacities of microorganisms to adapt to various environments can be laterally indicated by their growth rates. To the best of our knowledge, vibrios have the fastest growth rate of marine bacteria, and $V$. natriegens has been reported to have a maximal generation time of less than $10 \mathrm{~min}$ [73]. It has been considered that the number of rRNA operons may dictate the rate of ribosomes synthesis and the response to favorable changes in growth environments [74]. Therefore, the extremely rapid growth rate of vibrios may be explained by the much higher number of $16 \mathrm{~S}$ rRNA gene copies, which was, on average, more than three times of other compared marine bacteria in this study.

Bacteria have developed protective systems including R-M systems and CRISPR systems to defend external DNA invasion either by plasmids or virus [51]. Most vibrios in our analysis possessed at least one type of R-M system, and few of them also had CRISPR systems. These protective systems found in Vibrio genomes indicate their ability to defend themselves in marine environment and also suggest multiple encounters with foreign DNA.

Chitin metabolism is essential for carbon and nitrogen recycling in marine ecosystems. The capability of vibrios to use chitin provided possibilities for them to form biofilms on the surface of chitin, and the formation of these biofilms could affect many other biological functions [75]. Therefore, the chitinase family played an essential role in the growth and development of vibrios, which may explain the expansion of this family during the evolutionary process of the genus. We found that the numbers of chitinase genes in the genomes of Vibrio species (up to 9 and 16 in the small and large dataset, respectively) are much higher than that in the other compared marine bacteria (only two strains from Gammaproteobacteria had eight and two, respectively, while others did not carry chitinase genes), yet only two of which exist in the predicted MRCA of the genus Vibrio. As an expansion in size of a particular gene family suggested its importance for the adaptation of the species during evolution [76], the remarkable expansion of chitinase gene demonstrated the importance of the chitin catabolism process in vibrios.

The chitinolytic cascade in Vibrio species was attributed to a bunch of chitin degrading related genes. We found that $\sim 99 \%$ of Vibrio strains contained at least one chitinase coding gene in their genomes in the large dataset. However, a previous chitinase enzyme activity survey found that only $\sim 80 \%$ (37 out of 47 ) of Vibrio strains had the ability to grow on chitin substrates [8], which may be because some components required in the complex pathway of chitin-degrading were absent in the genomes. Studies on the nonpathogenic marine bacterium $V$. furnissii showed that chitin utilization was a complex process involving at least three steps: chitin sensing, attachment, and degradation [77], and it has been demonstrated that the lack of essential chitin degradation elements led to the loss of chitin-degrading activity [78]. Our analysis showed that the chitinase coding genes suffered HGT and duplication during the evolutionary history of vibrios, which may result in mutations in the sequences and followed by the loss of the chitin-degrading activity. In addition, the selective pressure calculation and phylogenic analysis of the gene chiA showed that the gene has undergone significant purifying selective force and may evolve along with most other protein families in vibrios. It could be deduced that there has been evolutionary pressure to make chiA conserve the ancestral state, and members of the chiA family may evolve independently by silent nucleotide substitution and may be subject to evolution by a birth-and-death process at the DNA level, which implies new genes are generated by gene duplication, and some duplicate genes may maintain in the genome, while others may be deleted or dysfunction through deleterious mutations [79]. A previous study showed that vibrios tend to acquire new genetic material more easily when they attach to chitin, and this process could help vibrios to grow in a previously hostile niche by slightly changing their genome content [80]. We noticed that the chitinase genes in Vibrio genomes presented random distribution patterns, and does not seem to correlate with phylogenetic relatedness, which may because the HGT or duplication events occurred in the Vibrio evolutionary history were randomly. Intriguingly, in the 19 Vibrio complete genomes, the two genomes of which does not possess genes encoding chitinase coincide with the two does not 
have R-M systems, suggesting that the R-M systems might be deleted from the genomes as the systems would be less useful for vibrios which lose chances to intake external DNA. This confirmed the important role of chitin metabolism in vibrios, and the fact that chitinase genes were abundant in Vibrio genomes potentially explained the reason why we found an extremely high rate of HGT occurring in vibrios.

\section{Conclusions}

This extensive comparative genomics study based on complete genomes revealed the evolutionary history and ecological adaptation strategies of the widely distributed marine group vibrios. The phylogenetic history of vibrios was inferred according to the core-genome tree, and the extremely gene gain events were found during the evolutionary process from 536 MYA, which may be largely driven by HGT. The steady core-genome and tremendous pan-genome made vibrios comprise necessary genetic materials and flexible accessory genes to be cosmopolitan. Most vibrios had defensive mechanisms including R-M systems and CRISPR systems to resist the invasion of foreign DNA. Furthermore, chitin-degrading related genes were carried in nearly all the Vibrio genomes, and the number of chitinase genes (up to 16) have extremely expanded from only two exist in the most recent ancestor of vibrios. Our study provided a comprehensive view of vibrios at genomic level, shedding light on the process that the evolution drives the abundant heterotrophic bacteria to be diversity and adapt to various marine environments.

\section{Additional files}

Additional file 1: Table S1. General features of the 20 complete genomes of Vibrio species analyzed. (DOCX $48 \mathrm{~kb}$ )

Additional file 2: Table S2. General features of the 13 marine bacteria complete genomes used to compare with Vibrio. (DOCX $34 \mathrm{~kb}$ )

Additional file 3: Figure S1. Pan and core plot of the 20 Vibrio species with complete genomes. Accumulation curves for (A) the number of genes in common or (B) total number of genes are plotted. The grey dots represent 10 different random input sequences of genomes, and the extrapolated limiting value for core genes is shown as a dashed line. Equations which can be used to fit the curves are shown above the plot respectively. (PDF $1023 \mathrm{~kb}$ )

Additional file 4: Figure S2. COGs classification of the specific genomes of $V$. rotiferianus B64D1 and V. mediterranei QT6D1. Designations of functional categories: [A] RNA processing and modification, [B] chromatin structure and dynamics, [C] energy production and conversion, [D] cell cycle control and mitosis, [E] amino acid metabolism and transport, [F] nucleotide metabolism and transport, [G] carbohydrate metabolism and transport, $[\mathrm{H}]$ coenzyme metabolism, [1] lipid metabolism, [J] translation, [K] transcription, [L] replication and repair, [M] cell wall/membrane/envelope biogenesis, [N] Cell motility, [O] post-translational modification, protein turnover, chaperone functions, [P] Inorganic ion transport and metabolism, [Q] secondary metabolites biosynthesis, transport and catabolism, [R] general functional prediction only, [S] function unknown, [T] signal transduction, [U] intracellular trafficking and secretion, $\mathrm{V}]$
Defense mechanisms, M] Extracellular structures, Y] nuclear structure, [Z] cytoskeleton. (PDF $822 \mathrm{~kb}$ )

Additional file 5: Figure S3. Pan genome tree. The tree was created based on the presence or absence of gene clusters in the 20 complete Vibrio genomes. The number at each node denotes the bootstrap value based on 1000 replicates. The color red and yellow are corresponding to the core genome tree, suggesting the discrepancy between core and pan genome trees. (PDF $194 \mathrm{~kb}$ )

Additional file 6: Figure S4. Neighbor-joining phylogenetic tree of the 20 vibrios genomes based on 16S rRNA gene. The number at each node denotes the bootstrap value based on 1000 replicates. S. denitrificans OS217 was used as the outgroup. Bar, 0.01 substitutions per site. (PDF 186 kb)

Additional file 7: Figure S5. Heatmap presentation of pairwise average nucleotide identity of the 19 Vibrio species with complete genomes. The genomes are hierarchical clustered according to the values of rows. The clusters present in blue and orange are corresponding to the core genome tree. (PDF $212 \mathrm{~kb}$ )

Additional file 8: Figure S6. Genes related to the chitin-degrading process in 20 vibrios with complete genomes. Each column indicates a chitin metabolism-related gene family, with the family name indicating the predicted function. The number in the box indicates the copy number of that gene family in the corresponding genome. (PDF $282 \mathrm{~kb}$ )

\section{Abbreviations}

ANI: Average nucleotide identity; HGT: Horizontal gene transfer; MA: Marine 2216E agar; ML: Maximum-likelihood; MLSA: Multilocus sequence analysis; MYA: Million years ago; ORF: Open reading frame; TCBS: Thiosulfate citrate bile salts sucrose

\section{Acknowledgements}

The authors thank professor Brian Austin from University of Stirling for critical reading of the manuscript.

\section{Funding}

This work was supported by the National Natural Science Foundation of China through grants 41730530, 41476112, 41521064 and 41506154.

\section{Availability of data and materials}

The data sets supporting the results of this article are available at the GenBank database under the accession numbers CP018311 to CP018312 and CP018308 to CP018310. All data used for the phylogenetic tree of this article are available in the TreeBase (http://treebase.org/treebase-web/home.html) repository, under the accession URL http://purl.org/phylo/treebase/phylows/ study/TB2:S22249. The materials generated during the current study are available from the corresponding author on reasonable request.

\section{Authors' contributions}

$\mathrm{X}-\mathrm{HZ}$ designed the study, interpreted the data and thoroughly revised the manuscript. $\mathrm{HL}$ and MY performed the bioinformatics analysis and wrote the manuscript. XW isolated the strains and sequenced the genomic DNA and analyzed the genomic data. All authors edited and approved the final version of the manuscript.

\section{Ethics approval and consent to participate}

Not applicable, as this study did not involve human or animal subjects, and the field sampling procedures met local guidelines.

\section{Consent for publication}

Not applicable.

\section{Competing interests}

The authors declare that they have no competing interests.

\section{Publisher's Note}

Springer Nature remains neutral with regard to jurisdictional claims in published maps and institutional affiliations. 


\section{Author details}

${ }^{1}$ College of Marine Life Sciences, Ocean University of China, 5 Yushan Road, Qingdao 266003, People's Republic of China. ${ }^{2}$ Laboratory for Marine Ecology and Environmental Science, Qingdao National Laboratory for Marine Science and Technology, Qingdao 266071, People's Republic of China. ${ }^{3}$ Institute of Evolution \& Marine Biodiversity, Ocean University of China, Qingdao 266003, People's Republic of China.

\section{Received: 9 April 2017 Accepted: 5 February 2018}

Published online: 13 February 2018

\section{References}

1. Thompson FL, lida T, Swings J. Biodiversity of vibrios. Microbiol Mol Biol Rev. 2004;68(3):403-31.

2. Colwell RR. Global climate and infectious disease: the cholera paradigm. Science. 1996;274(5295):2025-31.

3. Reen FJ, Almagro-Moreno S, Ussery D, Boyd EF. The genomic code: inferring Vibrionaceae niche specialization. Nat Rev Microbiol. 2006;4(9):697-704.

4. Kaneko T, Colwell RR. Ecology of Vibrio parahaemolyticus in Chesapeake Bay. J Bacteriol. 1973;113(1):24-32.

5. Simidu U, Taga N, Colwell RR, Schwarz JR. Heterotrophic bacterial flora of the seawater from the Nansei Shoto (Ryukyo Retto) area. Nippon Suisan Gakkaishi. 1980;46(4):505-10.

6. Eilers H, Pernthaler J, Glöckner FO, Amann R. Culturability and in situ abundance of pelagic bacteria from the North Sea. Appl Environ Microbiol. 2000:66(7):3044-51.

7. Yooseph S, Nealson KH, Rusch DB, McCrow JP, Dupont CL, Kim M, et al. Genomic and functional adaptation in surface ocean planktonic prokaryotes. Nature. 2010;468(7320):60-6.

8. Hunt DE, Gevers D, Vahora NM, Polz MF. Conservation of the chitin utilization pathway in the Vibrionaceae. Appl Environ Microbiol. 2008; 74(1):44-51

9. Gooday GW. The ecology of chitin degradation. In: Marshall KC, editor Advances in microbial ecology. Boston, MA: Springer US; 1990. p. 387-430.

10. Meibom KL, Li XB, Nielsen AT, Wu C-Y, Roseman S, Schoolnik GK. The Vibrio cholerae chitin utilization program. Proc Natl Acad Sci U S A. 2004:101(8):2524-9.

11. Defoirdt T, Darshanee Ruwandeepika HA, Karunasagar I, Boon N, Bossier P. Quorum sensing negatively regulates chitinase in Vibrio harveyi. Environ Microbiol Rep. 2010;2(1):44-9.

12. Li X, Roseman S. The chitinolytic cascade in Vibrios is regulated by chitin oligosaccharides and a two-component chitin catabolic sensor/kinase. Proc Natl Acad Sci U S A. 2004;101(2):627-31

13. Orikoshi H, Nakayama S, Miyamoto K, Hanato C, Yasuda M, Inamori Y, et al. Roles of four chitinases (ChiA, ChiB, ChiC, and ChiD) in the chitin degradation system of marine bacterium Alteromonas sp. strain 0-7. Appl Environ Microbiol. 2005;71(4):1811-5.

14. Markov EY, Kulikalova ES, Urbanovich LY, Vishnyakov VS, Balakhonov SV. Chitin and products of its hydrolysis in Vibrio cholerae ecology. Biochemistry (Mosc). 2015;80(9):1109-16.

15. Thompson CC, Vicente ACP, Souza RC, Vasconcelos ATR, Vesth T, Alves N, et al. Genomic taxonomy of vibrios. BMC Evol Biol. 2009;9(1):258.

16. Sawabe T, Ogura Y, Matsumura Y, Gao F, Amin A, Mino S, et al. Updating the vibrio clades defined by multilocus sequence phylogeny: proposal of eight new clades, and the description of Vibrio tritonius sp. nov. Front Microbiol. 2013;4(414)

17. Lukjancenko O, Ussery D. Vibrio chromosome-specific families. Front Microbiol. 2014;5(73)

18. Hazen TH, Pan L, Gu JD, Sobecky PA. The contribution of mobile genetic elements to the evolution and ecology of Vibrios. FEMS Microbiol Ecol. 2010;74(3):485-99.

19. Boucher Y, Cordero OX, Takemura A, Hunt DE, Schliep K, Bapteste E, et al. Local mobile gene pools rapidly cross species boundaries to create endemicity within global Vibrio cholerae populations. MBio. 2011;2(2)

20. Mardis E, McPherson J, Martienssen R, Wilson RK, McCombie WR. What is finished, and why does it matter. Genome Res. 2002;12(5):669-71.

21. Marmur J. A procedure for the isolation of deoxyribonucleic acid from micro-organisms. J Mol Biol. 1961;3(2):208-18.

22. Koren S, Walenz BP, Berlin K, Miller JR, Bergman NH, Phillippy AM. Canu: scalable and accurate long-read assembly via adaptive k-mer weighting and repeat separation. Genome Res. 2017;27(4)
23. Walker BJ, Abeel T, Shea T, Priest M, Abouelliel A, Sakthikumar S, et al. Pilon: an integrated tool for comprehensive microbial variant detection and genome assembly improvement. PLoS One. 2014;9(11):e112963.

24. Hunt M, Kikuchi T, Sanders M, Newbold C, Berriman M, Otto TD. REAPR: a universal tool for genome assembly evaluation. Genome Biol. 2013; 14(5):R47.

25. Brettin T, Davis JJ, Disz T, Edwards RA, Gerdes S, Olsen GJ, et al. RASTtk: a modular and extensible implementation of the RAST algorithm for building custom annotation pipelines and annotating batches of genomes. Sci Rep. 2015;5:8365.

26. Schattner P, Brooks AN, Lowe TM. The tRNAscan-SE, snoscan and snoGPS web servers for the detection of tRNAs and snoRNAs. Nucleic Acids Res. 2005:33(suppl 2):W686-W89.

27. Lagesen K, Hallin P, Rødland EA, Stærfeldt H-H, Rognes T, Ussery DW. RNAmmer: consistent and rapid annotation of ribosomal RNA genes. Nucleic Acids Res. 2007;35(9):3100-8.

28. Grissa I, Vergnaud G, Pourcel C. CRISPRFinder: a web tool to identify clustered regularly interspaced short palindromic repeats. Nucleic Acids Res. 2007:35(suppl_2):W52-7.

29. Urbanczyk H, Ast JC, Higgins MJ, Carson J, Dunlap PV. Reclassification of Vibrio fischeri, Vibrio logei, Vibrio salmonicida and Vibrio wodanis as Aliivibrio fischeri gen. nov., comb. nov., Aliivibrio logei comb. nov., Aliivibrio salmonicida comb. nov. and Aliivibrio wodanis comb. nov. Int J Syst Evol Microbiol. 2007:57(12):2823-9.

30. Gauthier G, Lafay B, Ruimy R, Breittmayer V, Nicolas JL, Gauthier M, et al. Small-subunit rRNA sequences and whole DNA relatedness concur for the reassignment of Pasteurella piscicida (Snieszko et al.) Janssen and Surgalla to the genus Photobacterium as Photobacterium damsela subsp. piscicida comb. nov. Int J Syst Evol Microbiol. 1995;45(1):139-44

31. Contreras-Moreira B, Vinuesa P. GET HOMOLOGUES, a versatile software package for scalable and robust microbial pangenome analysis. Appl Environ Microbiol. 2013:79(24):7696-701.

32. Kristensen DM, Kannan L, Coleman MK, Wolf YI, Sorokin A, Koonin EV, et al. A low-polynomial algorithm for assembling clusters of orthologous groups from intergenomic symmetric best matches. Bioinformatics. 2010;26(12):1481-7.

33. Li L, Stoeckert CJ, Roos DS. OrthoMCL: identification of ortholog groups for eukaryotic genomes. Genome Res. 2003;13(9):2178-89.

34. Katoh K, Standley DM. MAFFT multiple sequence alignment software version 7: improvements in performance and usability. Mol Biol Evol. 2013; 30(4):772-80.

35. Capella-Gutiérrez S, Silla-Martínez JM. Gabaldón T: trimAl: a tool for automated alignment trimming in large-scale phylogenetic analyses. Bioinformatics. 2009;25(15):1972-3.

36. Stamatakis A. RAxML version 8: a tool for phylogenetic analysis and postanalysis of large phylogenies. Bioinformatics. 2014;30(9):1312-3.

37. Abascal F, Zardoya R, Posada D. ProtTest: selection of best-fit models of protein evolution. Bioinformatics. 2005;21(9):2104-5.

38. Huson DH, Bryant D. Application of phylogenetic networks in evolutionary studies. Mol Biol Evol. 2006;23(2):254-67.

39. Sanderson MJ. Estimating absolute rates of molecular evolution and divergence times: a penalized likelihood approach. Mol Biol Evol. 2002;19(1):101-9.

40. Sanderson MJ. r8s: inferring absolute rates of molecular evolution and divergence times in the absence of a molecular clock. Bioinformatics. 2003; 19(2):301-2.

41. Falcon LI, Magallon S, Castillo A. Dating the cyanobacterial ancestor of the chloroplast. ISME J. 2010;4(6):777-83.

42. Bergthorsson $\mathrm{U}$, Ochman $\mathrm{H}$. Distribution of chromosome length variation in natural isolates of Escherichia coli. Mol Biol Evol. 1998;15(1):6-16.

43. Csüös M. Count: evolutionary analysis of phylogenetic profiles with parsimony and likelihood. Bioinformatics. 2010;26(15):1910-2.

44. Waack S, Keller O, Asper R, Brodag T, Damm C, Fricke WF, et al. Score-based prediction of genomic islands in prokaryotic genomes using hidden Markov models. BMC Bioinformatics. 2006;7(1):142

45. Goris J, Konstantinidis KT, Klappenbach JA, Coenye T, Vandamme P, Tiedje JM. DNA-DNA hybridization values and their relationship to whole-genome sequence similarities. Int J Syst Evol Microbiol. 2007;57(1):81-91.

46. Kolde R pheatmap: Pretty Heatmaps: R package version 1.0.8. Journal 2015, https://cran.r-project.org/web/packages/pheatmap/index.html.

47. Saitou N, Nei $M$. The neighbor-joining method: a new method for reconstructing phylogenetic trees. Mol Biol Evol. 1987;4(4):406-25. 
48. Kumar S, Stecher G, Tamura K. MEGA7: molecular evolutionary genetics analysis version 7.0 for bigger datasets. Mol Biol Evol. 2016;33(7):1870-4.

49. Yang Z. PAML 4: phylogenetic analysis by maximum likelihood. Mol Biol Evol. 2007;24(8):1586-91.

50. Barberán A, Casamayor EO. Global phylogenetic community structure and $\beta$-diversity patterns in surface bacterioplankton metacommunities. Aquat Microb Ecol. 2010;59:1-10.

51. Stern A, Sorek R. The phage-host arms race: shaping the evolution of microbes. BioEssays. 2011;33(1):43-51.

52. Tettelin H, Riley D, Cattuto C, Medini D. Comparative genomics: the bacterial pan-genome. Curr Opin Microbiol. 2008;11(5):472-7.

53. Dorsch M, Lane D, Stackebrandt E. Towards a phylogeny of the genus Vibrio based on 16S rRNA sequences. Int J Syst Evol Microbiol. 1992;42(1):58-63.

54. Sawabe T, Kita-Tsukamoto K, Thompson FL. Inferring the evolutionary history of vibrios by means of multilocus sequence analysis. J Bacteriol. 2007;189(21):7932-6.

55. Konstantinidis KT, Tiedje JM. Genomic insights that advance the species definition for prokaryotes. Proc Natl Acad Sci U S A. 2005;102(7):2567-72.

56. Kim M, Oh H-S, Park S-C, Chun J. Towards a taxonomic coherence between average nucleotide identity and 16S rRNA gene sequence similarity for species demarcation of prokaryotes. Int J Syst Evol Microbiol. 2014;64(2):346-51.

57. Ochman $\mathrm{H}$, Lawrence JG, Groisman EA. Lateral gene transfer and the nature of bacterial innovation. Nature. 2000;405(6784):299-304.

58. Svitil AL, Chadhain S, Moore JA, Kirchman DL. Chitin degradation proteins produced by the marine bacterium Vibrio harveyi growing on different forms of chitin. Appl Environ Microbiol. 1997;63(2):408-13.

59. Pichyangkura R, Kudan S, Kuttiyawong K, Sukwattanasinitt M, Aiba S-I. Quantitative production of 2-acetamido-2-deoxy-D-glucose from crystalline chitin by bacterial chitinase. Carbohydr Res. 2002;337(6):557-9.

60. Ohno T, Armand S, Hata T, Nikaidou N, Henrissat B, Mitsutomi M, et al. A modular family 19 chitinase found in the prokaryotic organism Streptomyces griseus HUT 6037. J Bacteriol. 1996;178(17):5065-70.

61. Thompson JR, Randa MA, Marcelino LA, Tomita-Mitchell A, Lim E, Polz MF. Diversity and dynamics of a North Atlantic coastal Vibrio community. Appl Environ Microbiol. 2004;70(7):4103-10.

62. Constantin de Magny G, Hasan N, Roche B. How community ecology can improve our understanding of cholera dynamics. Front Microbiol. 2014; 5(137): 137.

63. Reno ML, Held NL, Fields CJ, Burke PV, Whitaker RJ. Biogeography of the Sulfolobus islandicus pan-genome. Proc Natl Acad Sci U S A. 2009; 106(21):8605-10.

64. Qin Q-L, Xie B-B, Yu Y, Shu Y-L, Rong J-C, Zhang Y-J, et al. Comparative genomics of the marine bacterial genus Glaciecola reveals the high degree of genomic diversity and genomic characteristic for cold adaptation. Environ Microbiol. 2014;16(6):1642-53.

65. Giovannoni SJ, Tripp HJ, Givan S, Podar M, Vergin KL, Baptista D, et al. Genome streamlining in a cosmopolitan oceanic bacterium. Science. 2005; 309(5738):1242-5

66. Partensky F, Garczarek L. Prochlorococcus: advantages and limits of minimalism. Annu Rev Mar Sci. 2010;2(1):305-31.

67. Konstantinidis KT, Serres MH, Romine MF, Rodrigues JLM, Auchtung J, McCue L-A, et al. Comparative systems biology across an evolutionary gradient within the Shewanella genus. Proc Natl Acad Sci U S A. 2009; 106(37):15909-14.

68. Touchon M, Hoede C, Tenaillon O, Barbe V, Baeriswyl S, Bidet P, et al. Organised genome dynamics in the Escherichia coli species results in highly diverse adaptive paths. PLoS Genet. 2009;5(1):e1000344.

69. Tanner AR, Fuchs D, Winkelmann IE, Gilbert MT, Pankey MS, Ribeiro AM et al. Molecular clocks indicate turnover and diversification of modern coleoid cephalopods during the Mesozoic Marine Revolution. Proc Biol Sci. 2017; 284(1850):20162818.

70. Near TJ, Eytan Rl, Dornburg A, Kuhn KL, Moore JA, Davis MP, et al. Resolution of ray-finned fish phylogeny and timing of diversification. Proc Natl Acad Sci U S A. 2012;109(34):13698-703.

71. Ruby EG, McFall-Ngai MJ. Oxygen-utilizing reactions and symbiotic colonization of the squid light organ by Vibrio fischeri. Trends Microbiol. 1999;7(10):414-20.

72. Konstantinidis KT, Tiedje JM. Trends between gene content and genome size in prokaryotic species with larger genomes. Proc Natl Acad Sci U S A. 2004;101(9):3160-5.
73. Eagon RG. Pseudomonas natriegens, a marine bacterium with a generation time of less than 10 minutes. J Bacteriol. 1962;83(4):736-7.

74. Klappenbach JA, Dunbar JM, Schmidt TM. rRNA operon copy number reflects ecological strategies of bacteria. Appl Environ Microbiol. 2000;66(4):1328-33.

75. Flemming H-C, Wingender J. The biofilm matrix. Nat Rev Microbiol. 2010; 8(9):623-33.

76. Karlsson M, Stenlid J. Comparative evolutionary histories of fungal chitinases. In: Pontarotti P, editor. Evolutionary biology: concept, modeling, and application. Berlin, Heidelberg: Springer Berlin Heidelberg; 2009. p. 323-37.

77. Keyhani NO, Roseman S. Physiological aspects of chitin catabolism in marine bacteria. Biochim Biophys Acta-Gen Subj. 1999;1473(1):108-22.

78. Qin Q-L, Li Y, Zhang Y-J, Zhou Z-M, Zhang W-X, Chen X-L, et al. Comparative genomics reveals a deep-sea sediment-adapted life style of Pseudoalteromonas sp. SM9913. ISME J. 2011;5(2):274-84.

79. Nei M, Rooney AP. Concerted and birth-and-death evolution of multigene families. Annu Rev Genet. 2005;39:121-52.

80. Meibom KL, Blokesch M, Dolganov NA, Wu C-Y, Schoolnik GK. Chitin induces natural competence in Vibrio cholerae. Science. 2005;310(5755):1824-7.

\section{Submit your next manuscript to BioMed Central and we will help you at every step:}

- We accept pre-submission inquiries

- Our selector tool helps you to find the most relevant journal

- We provide round the clock customer support

- Convenient online submission

- Thorough peer review

- Inclusion in PubMed and all major indexing services

- Maximum visibility for your research

Submit your manuscript at www.biomedcentral.com/submit
Biomed Central 